\title{
Stochastic Multi-Scale Methods for Turbulent Flow Simulations
}

\author{
Stefan Heinz ${ }^{* 1}$ \\ ${ }^{1}$ University of Wyoming, Department of Mathematics, 1000 East University Avenue, Laramie, WY 82071, USA
}

\begin{abstract}
The use of currently available methods for turbulent flow simulations is faced with significant problems: one has the choice between efficient methods with relatively low predictive power (which require evidence in each case) or relatively accurate but very expensive methods. The unification of these methods would be very helpful for a better understanding of the generality of modeling assumptions, and to make an optimal use of characteristic advantages of models. Previously, this problem was addressed on the basis of deterministic equations, which resulted in many different heuristic interpolation procedures. These problems can be solved by addressing the question considered at the more general level of stochastic equations: in this way it is possible to obtain consistent stochastic multi-scale methods which imply corresponding deterministic equations.
\end{abstract}

(c) 2006 WILEY-VCH Verlag GmbH \& Co. KGaA, Weinheim

\section{Large-scale methods for turbulent flow simulations}

The numerical integration of basic equations of fluid and thermodynamics represents a unique tool for studying fundamental mechanisms of turbulent flows, but the computational costs related to such direct numerical simulation (DNS) do not allow applications to most of the engineering and environmental flows [1]-[2]. In order to overcome this problem one has to reduce the computational requirements by modeling at least a part of the spectrum of turbulent motions.

The simplest way to address this question is to use an ensemble average to filter the basic equations, which results in deterministic Reynolds-averaged Navier-Stokes (RANS) equations [2]-[4]. According to the ergodic hypothesis, however, such ensemble-averaged fluid dynamic variables may be seen as spatial averages where the filter width is large compared to the characteristic length scale of turbulent eddies. In other words, all the spectrum of turbulent motions has to be modeled by developing in this way equations for the dynamics of ensemble-averaged fluid dynamic variables. The latter fact may result in significant problems which are illustrated in Fig. 1a. The transport equation for the dissipation rate (which determines the scale of turbulent motions) is essentially characterized by the standardized source rate $\mathrm{S}$. The majority of all turbulence models considers $\mathrm{S}$ as a linear function of the production-to-dissipation ratio $\mathrm{p}$ of turbulent kinetic energy [2]-[3]. The comparison with DNS data shown in Fig. 1a reveals, however, that this standard model can only indicate the general trend of variations of $\mathrm{S}$ (with a significant inaccuracy) [5]. It is also known that various RANS models are needed for different flows.

The use of stochastic equations which explain both dynamics of mean variables and turbulent fluctuations represents an attractive way to overcome important problems of RANS equations. Such probability density function (PDF) methods (which may be constructed by rescaling stochastic methods for molecular motion [6]) offer many advantages: their use provides closures for correlations of turbulent fluctuations which appear as unknowns in RANS equations, and important source terms (chemical source terms) can be involved without any need for closure assumptions [2], [7]-[8]. However, such PDF methods also suffer from the reference to ensemble means: equations for complex flows may well become rather complicated [8], and all these methods require scale information such that they are faced with the problem illustrated in Fig. 1a.
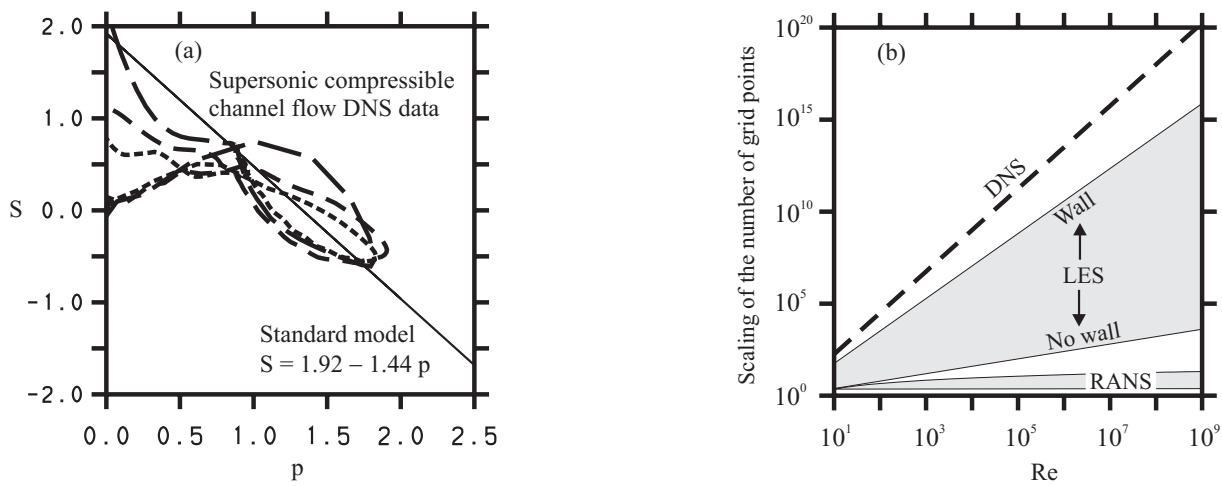

Fig. 1 (a) DNS data of the standardized source rate $S$ in the dissipation rate equation are shown against the production-todissipation ratio $\mathrm{p}$ of turbulent kinetic energy for three different Reynolds and Mach numbers [5]. Figure (b) illustrates the scaling of the number of grid points related to the use of different methodologies in dependence on the Reynolds number Re.

* Corresponding author: e-mail: heinz@ uwyo.edu, Phone: +01 307 766 4203, Fax: +01 3077666838 


\section{Small-scale methods for turbulent flow simulations}

A natural way to overcome the problems described above is to replace the coarse ensemble averages applied in RANS equations by spatially filtered variables, which provides large eddy simulation (LES) methods [9]-[10]. The fact that modeling is only applied to small-scale processes is very helpful. LES equations are simpler than RANS equations such that modeling issues related to the use of RANS methods for complex flows can be avoided. Usually one also finds that LES have much more predictive power than RANS simulations.

The significant disadvantage of LES methods is given by the fact that their computational costs are much higher than corresponding costs related to the use of RANS methods: see the illustration in Fig. 1b. One observes that the costs of LES for wall-bounded flows may even become close to the costs required to perform DNS. The suitability of LES methods for the solution of questions of practical relevance is, therefore, limited: such simulations become infeasible for high-Reynolds number flows such as occur in aeronautical and meteorological applications.

In correspondence to the generalization of RANS methods by stochastic PDF methods, LES methods can be generalized by stochastic filter density function (FDF) methods [11]. Such FDF methods offer all the advantages of PDF methods [11]-[13]: they explain the dynamics of small-scale turbulent processes, and chemical reactions can be involved without any need for closure assumptions. However, the costs of FDF methods are much higher than the costs required for LES: FDF simulations are six times less expensive than DNS, but they may require 15-30 times more effort than LES methods [11].

\section{Stochastic multi-scale methods for turbulent flow simulations}

Due to the high computational requirements related to the use of FDF and LES methods, at least the simulation of highReynolds number wall-bounded flows requires, therefore, combinations of FDF and PDF, or LES and RANS methods. The use of consistent combinations appears to be helpful to perform accurate computations. Thus, there is a need for the development of unified turbulence models that may be used depending on the resolution as FDF or PDF, LES or RANS methods. From a more general point of view, the development of such methods appears to be helpful to improve the efficiency and accuracy of turbulence simulations: accurate FDF and LES calculations could be applied to assess and improve the performance of more efficient PDF and RANS calculations. Also from a theoretical point of view, the development of unified turbulence models is relevant: a common modeling strategy may well contribute to model developments.

However, the construction of such unified models turned out to represent a non-trivial problem. Previously presented suggestions addressed this problem by considering the question of how it is possible to find a generalized stress tensor that recovers the Reynolds stress and subgrid-scale stress tensors in limits [8]-[9], [14]-[15]. The stress tensor is the result of a variety of physical processes. It is, therefore, impossible to find support for the construction of a generalized stress tensor by taking reference to a simple physical explanation of scale effects. The only way to develop generalized stress tensor models is then given by the application of heuristic interpolation procedures between RANS and LES limits. Many different suggestions were presented for that, and the assessment of differences between such suggestions turned out to represent a complicated problem. A general formal solution to the question considered was presented recently by Germano in conjunction with an analysis of all the problems related to the application of this approach [15].

A way to overcome this problem is to consider this question at the more general level of stochastic equations [16]. The significant advantage of this approach is the separate consideration of the dynamics of velocity fluctuations and their characteristic time scales. An appropriate generalization of time scales used in FDF and PDF methods then enables the derivation of a stochastic equation which recovers FDF and PDF methods in limit cases of a small and large filter width, respectively. Corresponding LES and RANS models are then implied as a consequence of the unified stochastic model. A detailed discussion of this approach (including the consideration of questions regarding the complexity of the method applied and relation between filtering in space and ensemble averaging) may be found elsewhere [16].

\section{References}

[1] P. Moin and K. Mahesh, Annu. Rev. Fluid Mech. 30, 539-578 (1998).

[2] S. B. Pope, Turbulent Flows (Cambridge University Press, Cambridge, 2000).

[3] D. C. Wilcox, Turbulence Modeling for CFD (Second edition, DCW Industries, Inc., 1998).

[4] P. A. Durbin and B. A. Petterson, Statistical Theory and Modeling for Turbulent Flows (John Wiley and Sons, New York, 2001).

[5] S. Heinz, Turbulent supersonic channel flow: Direct numerical simulation and modeling, AIAA Journal 44, in press (2006).

[6] S. Heinz, Physical Review E 70, 036308 (2004).

[7] R. O. Fox, Computational Models for Turbulent Reacting Flows (Cambridge University Press, Cambridge, 2003).

[8] S. Heinz, Statistical Mechanics of Turbulent Flows (Springer-Verlag, Berlin, 2003).

[9] P. Sagaut, Large Eddy Simulation for Incompressible Flows (Second edition, Springer-Verlag, Berlin, 2002).

[10] M. Lesieur, O. Metais and P. Comte, Large-Eddy Simulations of Turbulence (Cambridge University Press, Cambridge, 2005).

[11] P. Givi, AIAA Journal 44, 16-23 (2006).

[12] S. Heinz, Flow, Turb. Combust. 70, 153-181 (2003).

[13] S. Heinz, Phys. Fluids 17, 099101 (2005).

[14] C. G. Speziale, AIAA Journal 36, 173-184 (1998).

[15] M. Germano, Theor. Comp. Fluid Dyn. 17, 225-231 (2004)

[16] S. Heinz, Unified Turbulence Models for LES and RANS, FDF and PDF Simulations, Theor. Comp. Fluid Dyn. 20, submitted (2006). 